\title{
Gabies - Campagne de 2019 du musée du Louvre
}

\section{Steve Glisoni}

\section{OpenEdition \\ Journals}

\section{Édition électronique}

URL : http://journals.openedition.org/cefr/4672

DOI : $10.4000 /$ cefr.4672

ISSN : 2282-5703

\section{Éditeur}

École française de Rome

Référence électronique

Steve Glisoni, « Gabies - Campagne de 2019 du musée du Louvre », Chronique des activités

archéologiques de l'École française de Rome [En ligne], Italie centrale, mis en ligne le 25 novembre 2020, consulté le 04 décembre 2020. URL : http://journals.openedition.org/cefr/4672 ; DOI : https://doi.org/ $10.4000 /$ cefr.4672

Ce document a été généré automatiquement le 4 décembre 2020.

(c) École française de Rome 


\title{
Gabies - Campagne de $2019 \mathrm{du}$ musée du Louvre
}

\author{
Steve Glisoni
}

\section{NOTE DE L'AUTEUR}

Ce programme de recherche issu d'un partenariat entre le Musée du Louvre et la Soprintendenza Speciale Archeologia, Belle Arti e Paesaggio di Roma est financé par la Société des Amis du Louvre. Il est également réalisé en collaboration avec l'Inrap et en association avec l'École française de Rome. $\mathrm{N}^{\circ}$ de concession MIBACT 12395, du 02/05/2019.

L'équipe de 2019 était constituée de : Steve Glisoni (archéologue, Inrap, Musée du Louvre), Isabelle Hasselin (chargé d'études documentaires principale - responsable du centre d'études et de documentation au département des AGER), Didier Laroche (architecte historien, École nationale supérieure d'architecture de Strasbourg, Université de Strasbourg), Carlo Monda (architecte), Mehdi Belarbi (archéologue topographe, Inrap), Sophie Borg (archéologue, Service archéologique de la Ville de Lyon), Pierre Broutin (archéologue, Inrap), Samuel Desoutter (archéologue, Inrap), Sandra Zanella (archéologue - chercheuse associée au Laboratoire Archéologie des Sociétés Méditerranéennes UMR 5140 -CNRS), Aloïs Corona (archéologueanthropologue, Service archéologique EPI 78-92), Cristina Molari (céramologue), Nadia Cipolli (céramologue), Sophie Barat (archéologue, spécialiste du mobilier en verre), Christian Décamps (photographe, département des Antiquités égyptiennes du musée du Louvre), Grichka Bredow (archéologue, Service archéologique de la Ville de Lyon), Hugo Cador (archéologue), Camille Colonna (archéologue - anthropologue, Inrap), Laura Favreau (Gestionnaire des bases de données, DAGER Musée du Louvre), Fred Métais (archéologue, Inrap), Antoine Valois (archéologue, Inrap), Agnès Scherer (documentaliste scientifique, DAGER Musée du Louvre), Marjolaine Bénaich (étudiante en archéologie, École normale supérieure), Chloé Damay (doctorante en archéologie, Université de Rennes 2), Heidelinde Hupfer (doctorante en archéologie, École du 
Louvre), Clara Michaud (étudiante en archéologie, École du Louvre), Marta de Paola (étudiante en histoire de l'art, Università di Bologna, Alma Mater Studiorum), Clara Prats (étudiante en archéologie, Université Toulouse II, Jean Jaurès).

Nous remercions vivement la dott.ssa Daniela Porro, Soprintendente Speciale, ainsi que la dott.ssa Chiara Andreotti et le dott. Rocco Bochicchio, responsables du site de Gabies.

1 La campagne de fouille de 2019 s'est déroulée du $1^{\mathrm{er}}$ au 26 juillet 2019. Les recherches se sont concentrées dans le secteur A (fig. 1) sur la fouille du passage latéral oriental (aditus - espace A6) situé entre la scène et la cavea. Toute la séquence stratigraphique depuis le banc rocheux jusqu'au niveau de destruction de l'UA $1^{1}$ a ainsi été mise en évidence. L'écrêtement de la berme nord également a été l'occasion de dégager toute la partie supérieure du mur de soutènement de la cavea (MR 604). Dans le secteur B, nous avons achevé la fouille des pièces de l'UA $2^{2}$, à l'exception des pièces B2 et B8. Les niveaux de démolition de ces différentes pièces ont livré une importante quantité de mobilier archéologique, notamment de nombreux fragments d'enduits peints et de stucs d'époque impériale.

Figure 1 - Plan général des fouilles du musée du Louvre à Gabies (2013-2019).

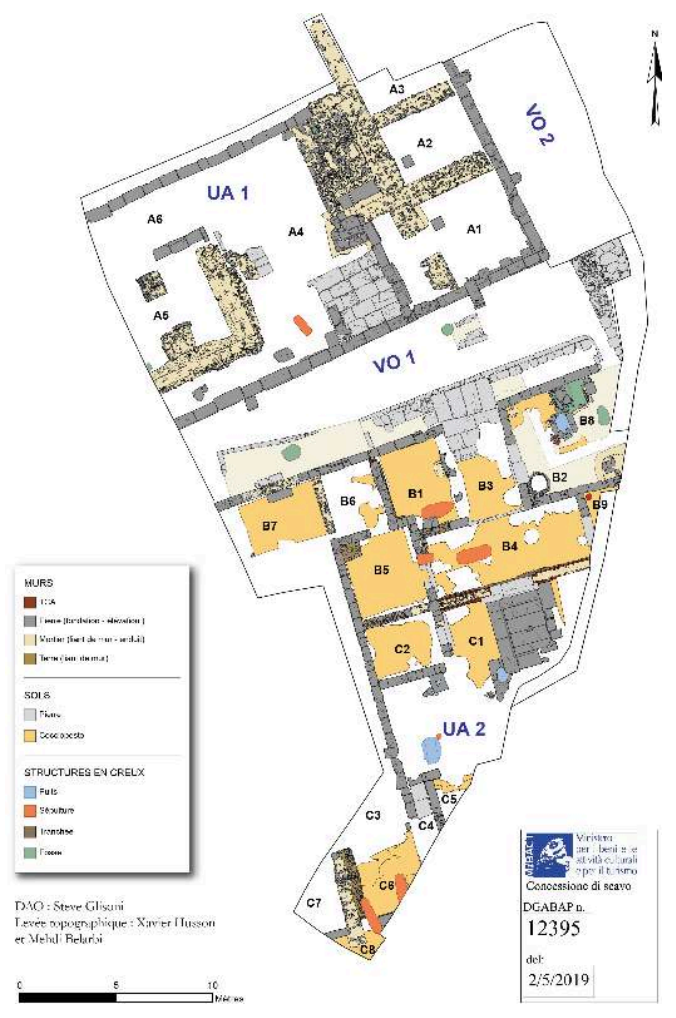

\section{La domus UA 2}

2 La fouille a concerné les pièces nord-est et nord-ouest de la domus (bâtiment UA 2; fig. 2) : les pièces B7 et B6 qui bordent la rue est-ouest VO 1, la pièce B5 qui s'ouvre sur l'atrium et les pièces B2/B8 situées dans l'angle nord-est de l'édifice, à l'intersection avec la rue nord-sud VO 2. 
Figure 2 - Plan général de la domus UA 2.

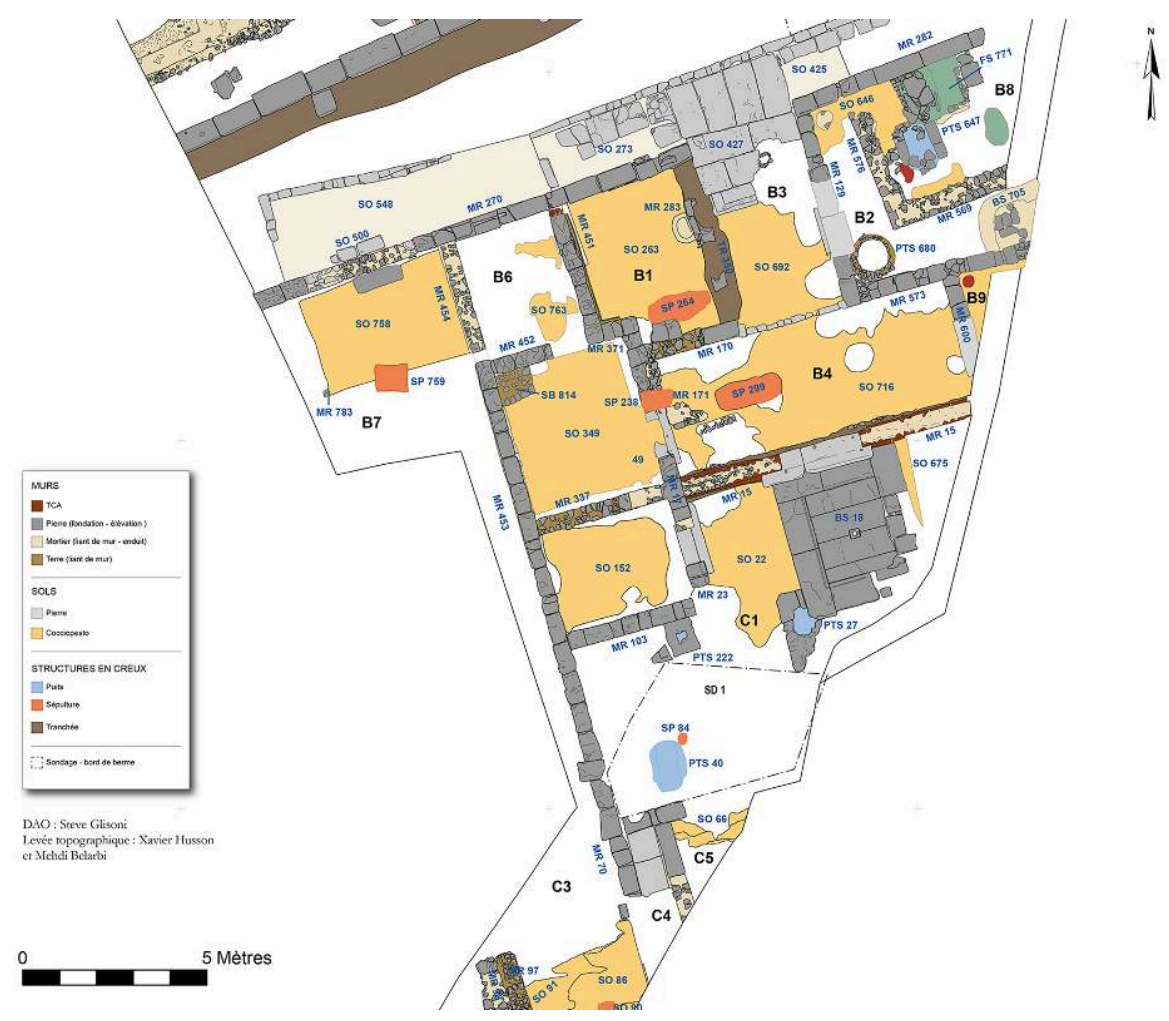

3 La pièce $\mathrm{B} 7$, dont les dimensions exactes restent à déterminer, a été fouillée sur environ $15 \mathrm{~m}^{2}$ (fig. 3). Le sol de la pièce est constitué d'une dalle de béton gris-beige présentant des traces de peinture rouge (SO 758). Il a été directement coulé sur un remblai de terre. Une marche en tuf de Gabies est placée en face de l'entrée de la pièce donnant sur la rue ${ }^{3}$, le sol étant situé $0,20 \mathrm{~m}$ plus bas $(64,12 \mathrm{~m}$ d'altitude) que le trottoir $(64,32 \mathrm{~m})$. Cette entrée a été par la suite rebouchée. Le mur oriental de la pièce, conservé sur $0,40 \mathrm{~m}$ d'élévation, présente la base d'un décor pariétal avec des panneaux à motif imitant le marbre avec des inter panneaux bleus et noirs. L'ensemble, encore à l'étude, peut pour l'instant être daté du $\mathrm{I}^{\mathrm{er}} \mathrm{s}$. apr.J.-C. Il a fait l'objet de mesures de conservation in situ. La pièce est colmatée par un remblai de démolition (US 757) qui a livré un abondant matériel archéologique, notamment de nombreux fragments d'enduits peints provenant du décor pariétal. 
Figure 3 - Le sol maçonné SO 758 dans la pièce B7 (G. Bredow).

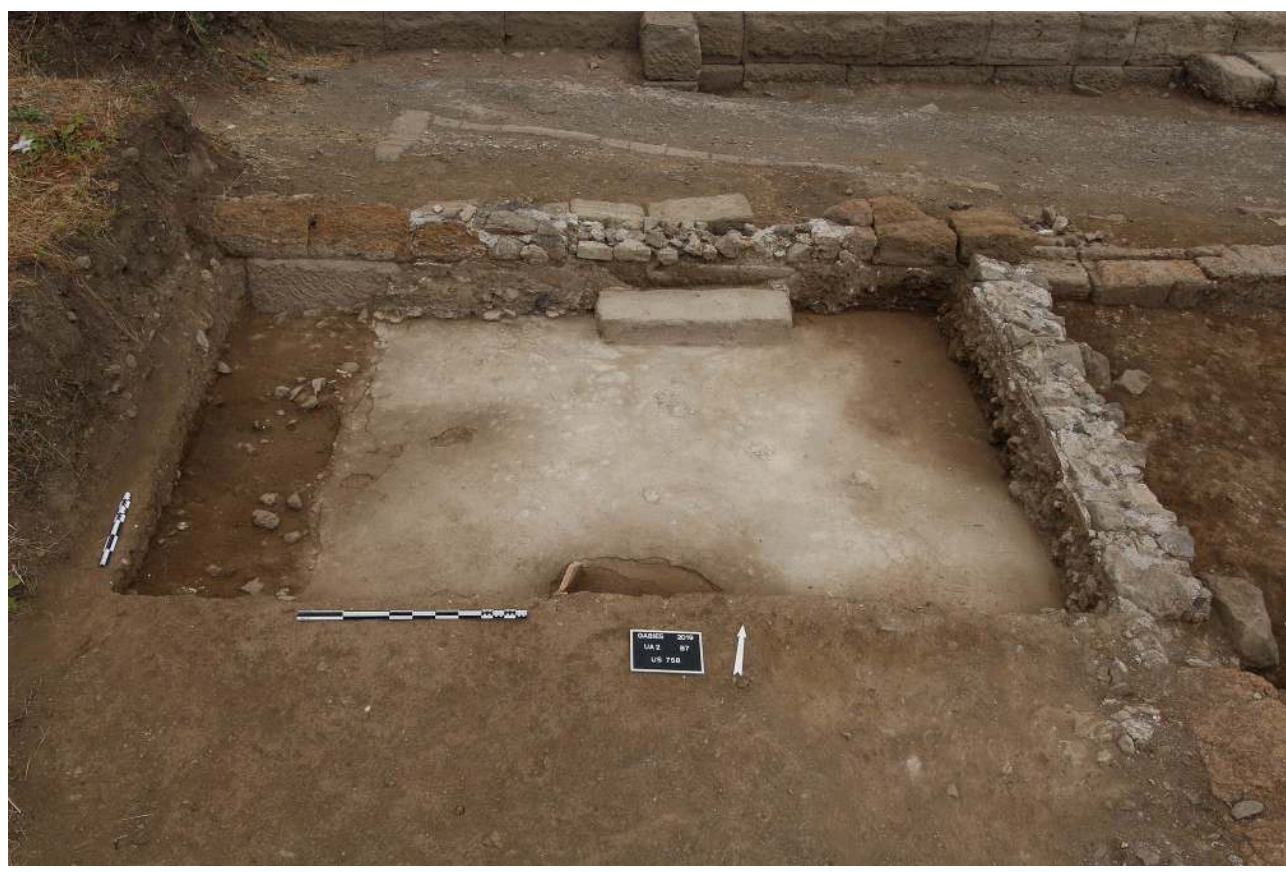

4 Une tombe à incinération (SP 759) aménagée avec un coffrage de tuiles disposées en bâtière est par la suite installée dans la pièce après l'abandon du bâtiment (fig. 4). Le coffrage est de forme carrée et mesure $0,70 \mathrm{~m}$ de côté pour $0,40 \mathrm{~m}$ de profondeur.

Figure 4 - Le coffrage en tuiles de la sépulture SP 759 (A. Corona).

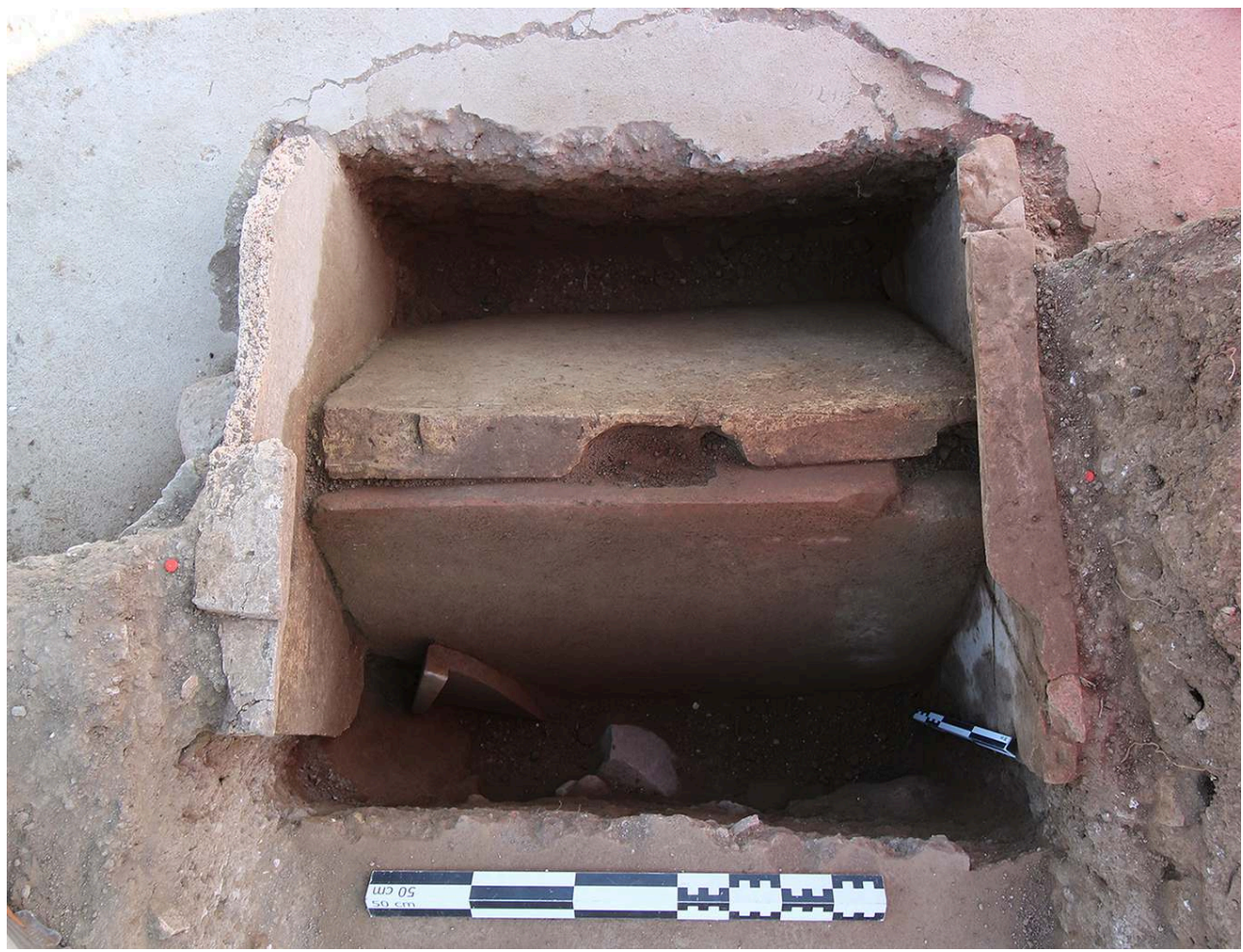

5 La pièce B5 possède une surface de $14,80 \mathrm{~m}^{2}$ et a été fouillée dans sa totalité (fig. 5). Son sol est constitué d'une dalle en béton de tuileau blanc-beige moyen à rouge-orangé 
clair. Une structure maçonnée quadrangulaire (SB 814 ; fig. 6) a été mise au jour dans l'angle nord-ouest de la pièce. Elle mesure $0,94 \mathrm{~m}$ de long pour $0,74 \mathrm{~m}$ de large et $0,40 \mathrm{~m}$ de hauteur. Le sol de la pièce $\mathrm{B} 5$ est situé à $63,75 \mathrm{~m}$, c'est-à-dire légèrement plus bas que le sol de la pièce B7 et que celui de la pièce adjacente B6 $(64,10 \mathrm{~m})$. L'absence d'un emmarchement au niveau de l'ouverture séparant les deux pièces peut être le fait soit d'une spoliation, soit de l'utilisation d'un matériau périssable.

Figure 5 - Vue de la pièce B5 depuis le nord (M. Belarbi).

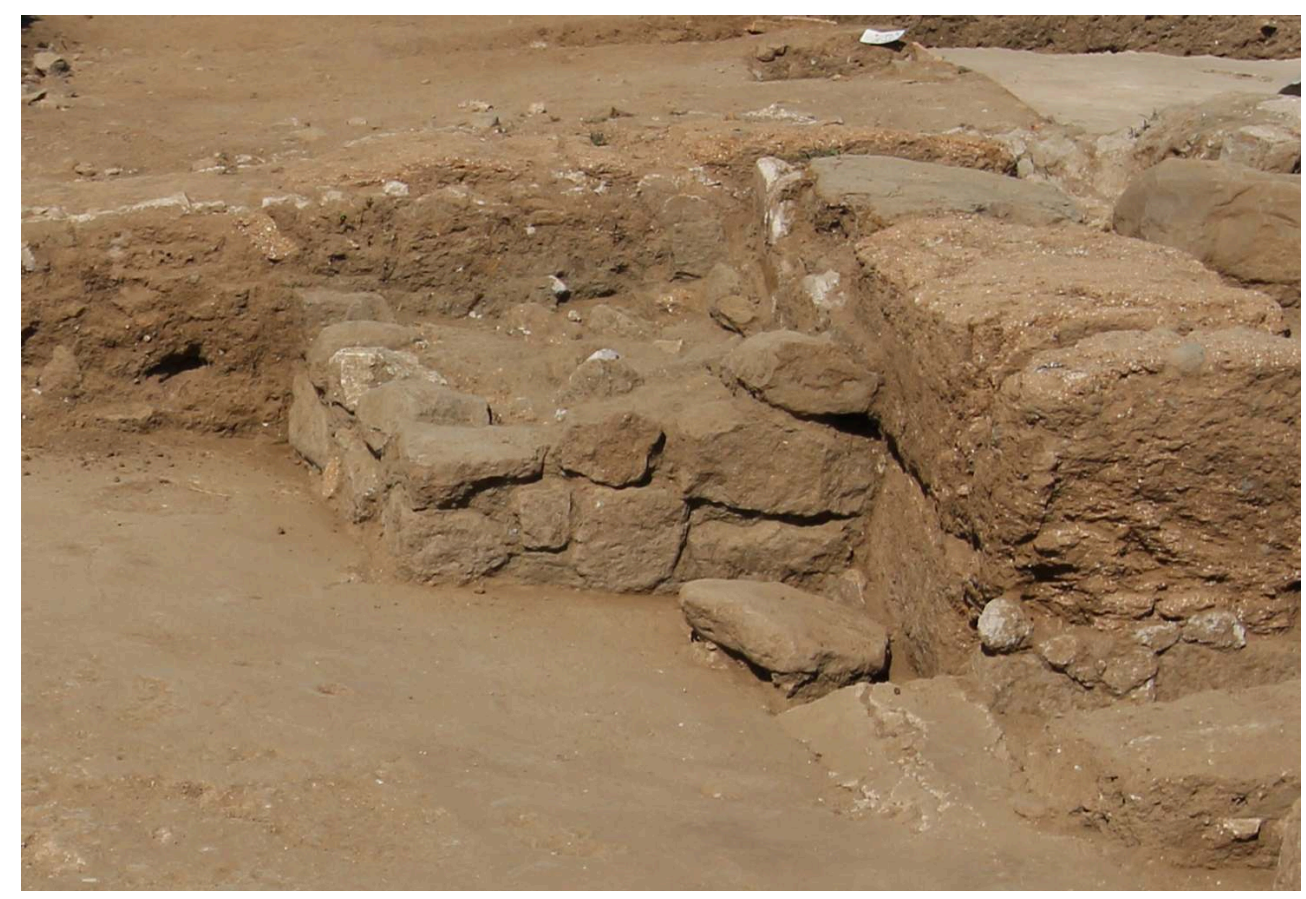

Figure 6 - La structure maçonnée SB 814 (M. Belarbi).

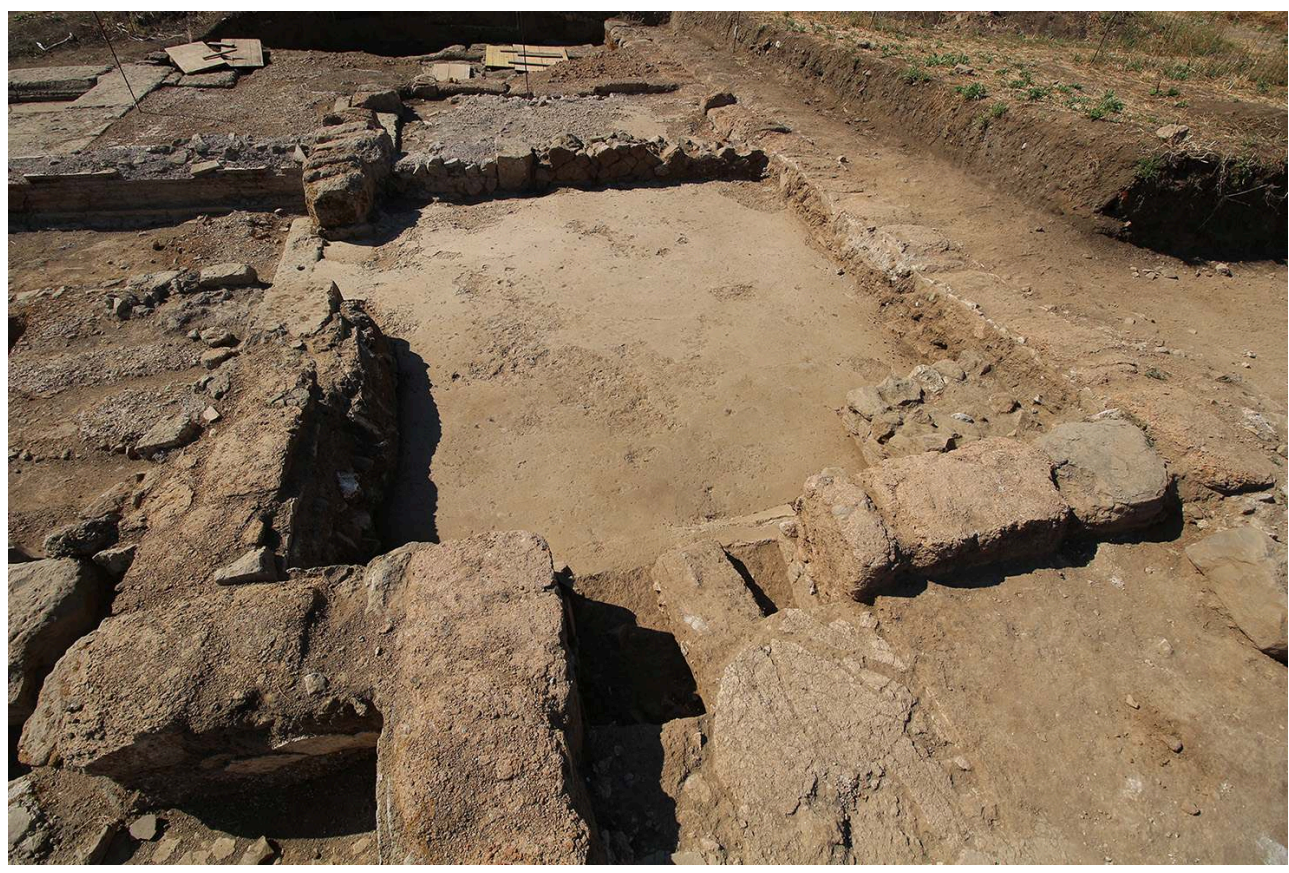


6 Les pièces B5 et B6 étaient également comblées par des remblais de démolition (US 102, 761 et 449) riches en mobilier archéologique comprenant des fragments d'enduits peints se rapportant à un décor peut-être de troisième style pompéien ainsi que des éléments de stucs et de lapidaires moulurés (fig. 7).

Figure 7 - Le remblai de démolition (US 761) de la pièce B6 (S. Zanella).

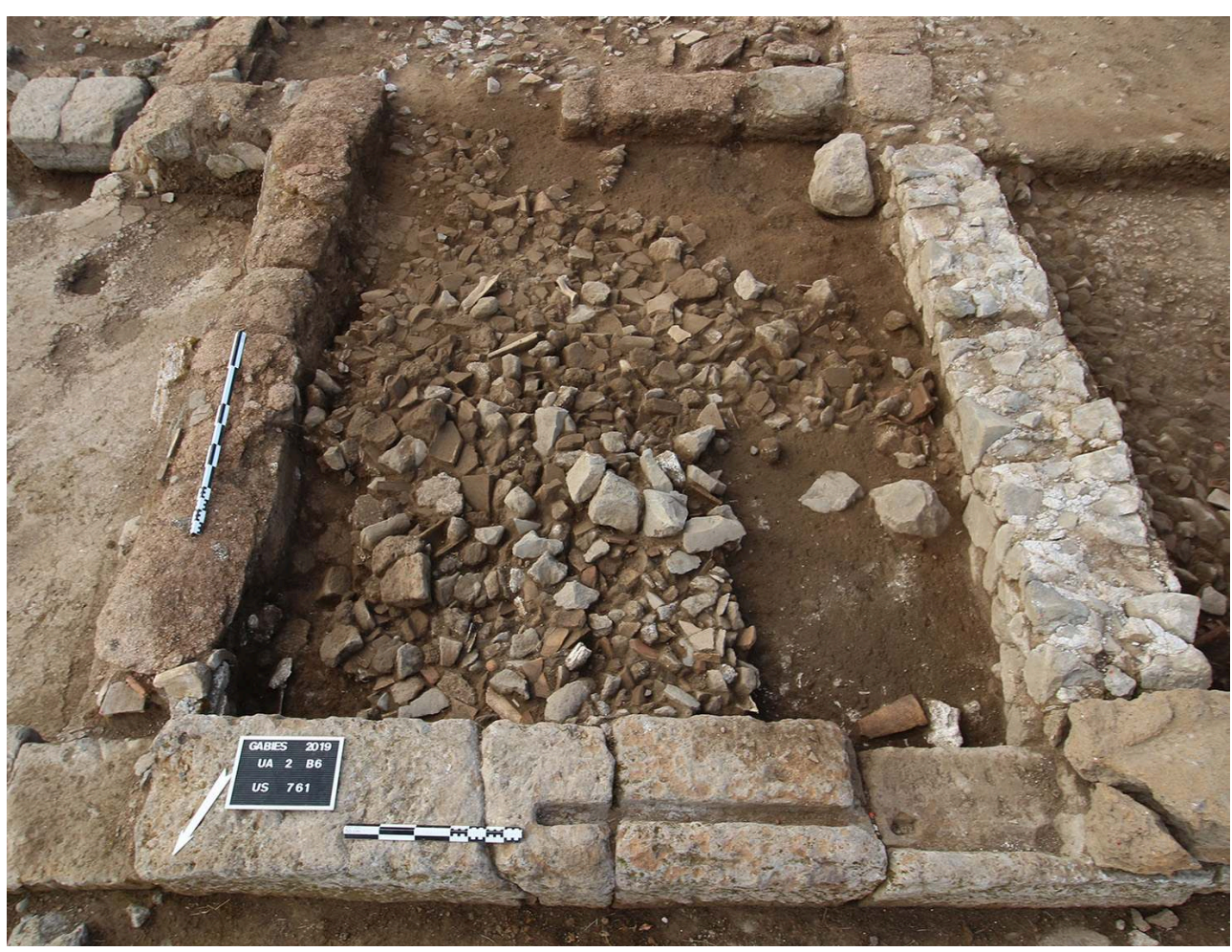

7 Les remblais de destruction de ces trois pièces sont datés entre la seconde moitié du II s. et la première moitié du III $\mathrm{e}$ s. apr. J.-C.

8 Dans le secteur oriental, seule la partie nord (pièces B2 et B8 et très partiellement la pièce B9) a pu être ouverte, le reste de la domus se trouvant sous l'actuel chemin de desserte du site (fig. 8). La pièce $\mathrm{B} 2 / \mathrm{B}^{4}$ présente une stratification plus complexe que les pièces $\mathrm{du}$ secteur ouest. Elle accueille notamment des aménagements dont la fonction exacte reste à déterminer pour certains. La découverte de structures hydrauliques (PTS 680, 647 et BS 705) suggère la présence d'une activité (peut-être artisanale) nécessitant un accès et une utilisation fréquente de l'eau sans que l'on puisse à l'heure actuelle en préciser la nature. 
Figure 8 - Vue zénithale du secteur nord-est de la domus - pièces B2 et B8 (M. Belarbi).

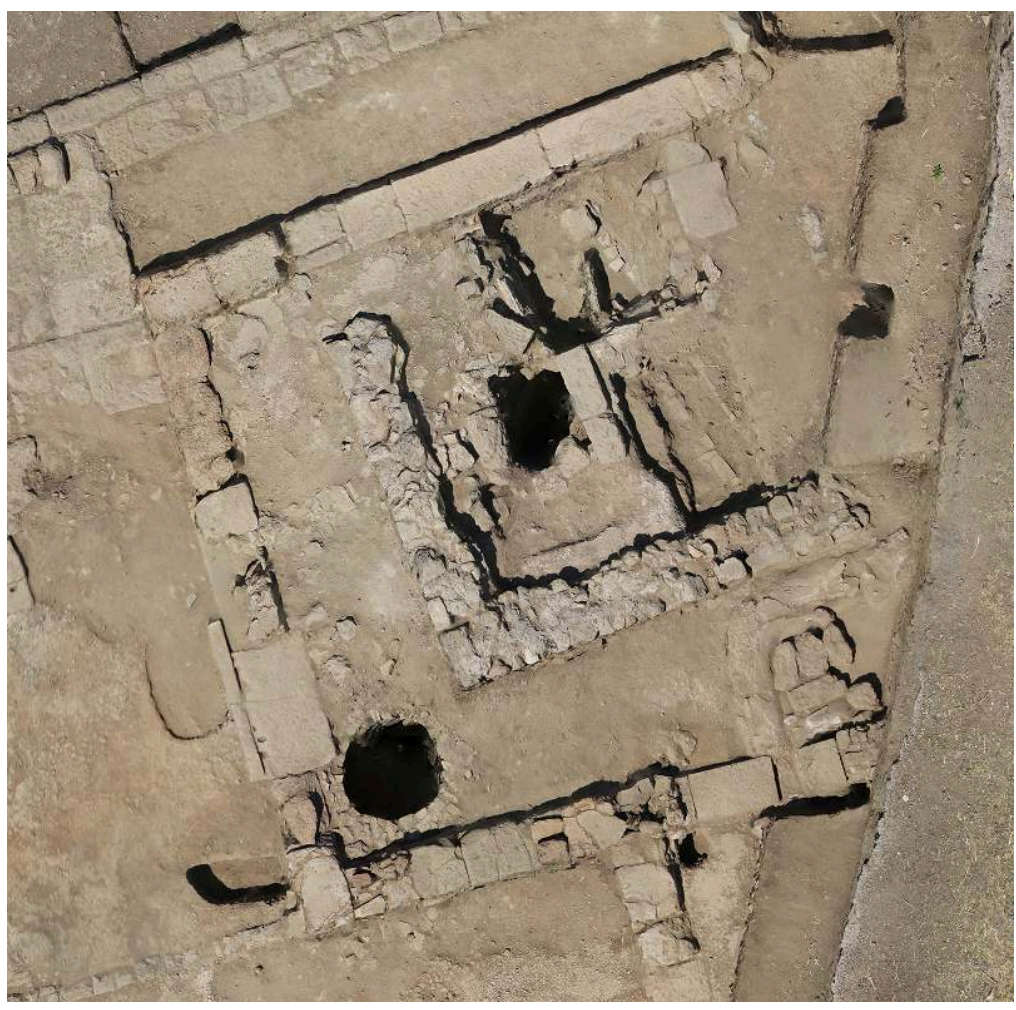

9 La fouille du puits PTS 680 , localisé dans l'angle sud-ouest de la pièce B2 près du seuil d'entrée, a mis en évidence que celui-ci est relié à deux galeries souterraines (cuniculi) voûtées creusées dans le tuf (fig. 9). Le haut du puits est maçonné tandis que le reste du conduit est directement creusé dans le banc rocheux. La maçonnerie qui couronne le puits est montée à la terre et mesure $0,25 \mathrm{~m}$ de large, son diamètre externe est de $1,24 \mathrm{~m}$ et son diamètre interne, c'est-à-dire l'ouverture du puits, est de 0,86 m. Elle est constituée de deux à quatre assises irrégulières en petit appareil rectangulaire employant des moellons de tuf (lapis Gabinus et tuf orangé) mesurant environ $0,10 \mathrm{~m}$ de côté en moyenne et dont la face de parement est équarrie. On trouve aussi quelques fragments de tuiles. Le blocage externe est composé de blocs de tuf gris de tailles différentes (jusqu'à $0,20 \mathrm{~m}$ de large). À l'ouest la maçonnerie présente quatre assises, au nord trois, à l'est et au sud deux assises. Cette irrégularité est due au pendage est-ouest du banc rocheux. 
Figure 9 - Vue en coupe depuis l'est du puits PTS 680 (photogrammétrie M. Belarbi, traitement photographique $C$. Décamps).

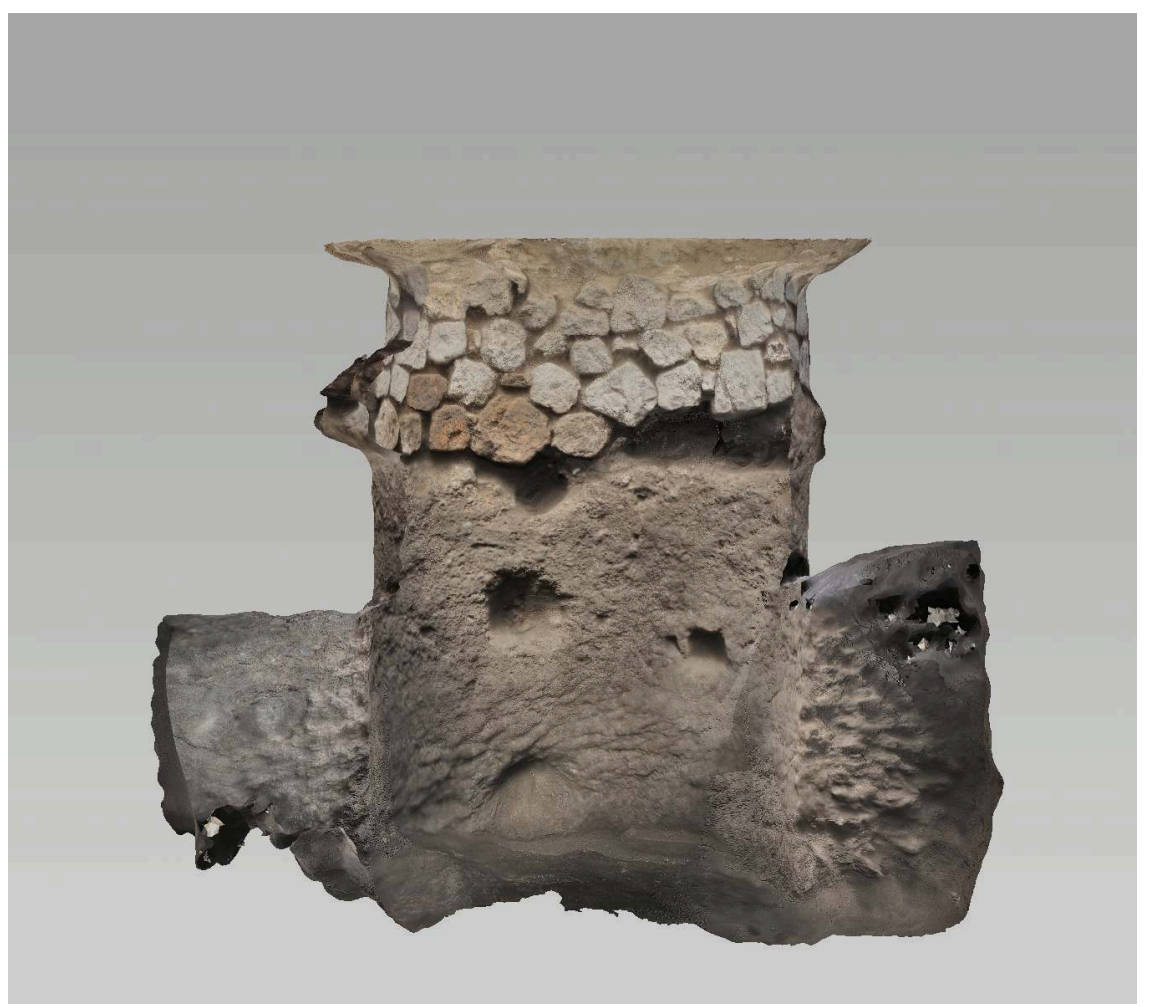

10 Les galeries se trouvent à environ $0,60 \mathrm{~m}$ de profondeur et ont été dégagées sur une hauteur de $0,80 \mathrm{~m}$. La première galerie suit une orientation nord-sud, identique à la rue VO 2 et pourrait être reliée à la citerne de l'impluvium, tandis que la seconde part vers le nord-est. Elle est reliée à un regard situé à moins de $5 \mathrm{~m}$ de distance dans la pièce $\mathrm{B} 8$ (PTS 647) et continue en direction de la rue VO 1. Le puits a été fouillé sur une profondeur de $1,45 \mathrm{~m}^{5}$ et son colmatage a livré du mobilier céramique permettant de dater son abandon entre la deuxième moitié du II ${ }^{\mathrm{e}}$ s. et le III ${ }^{\mathrm{e}}$ s. apr. J.-C.

D'autre part, un tronçon de caniveau (CN 794) a priori antérieur à la construction de la domus et construit avec des éléments de terres cuites architecturales liés à la terre, a été mis au jour sous des remblais de terrassement liés à une restructuration de cette partie de l'édifice à l'époque impériale, probablement durant le I ${ }^{\mathrm{er}} \mathrm{s}$. apr. J.-C. (fig. 10). Un angle de mur constitué de MR 569 et MR 576 vient ensuite diviser cet espace entre le $\mathrm{II}^{\mathrm{e}}$ et le III $\mathrm{e}$ s. apr. J.-C. 
Figure 10 - La canalisation CN 794 (H. Hupfer).

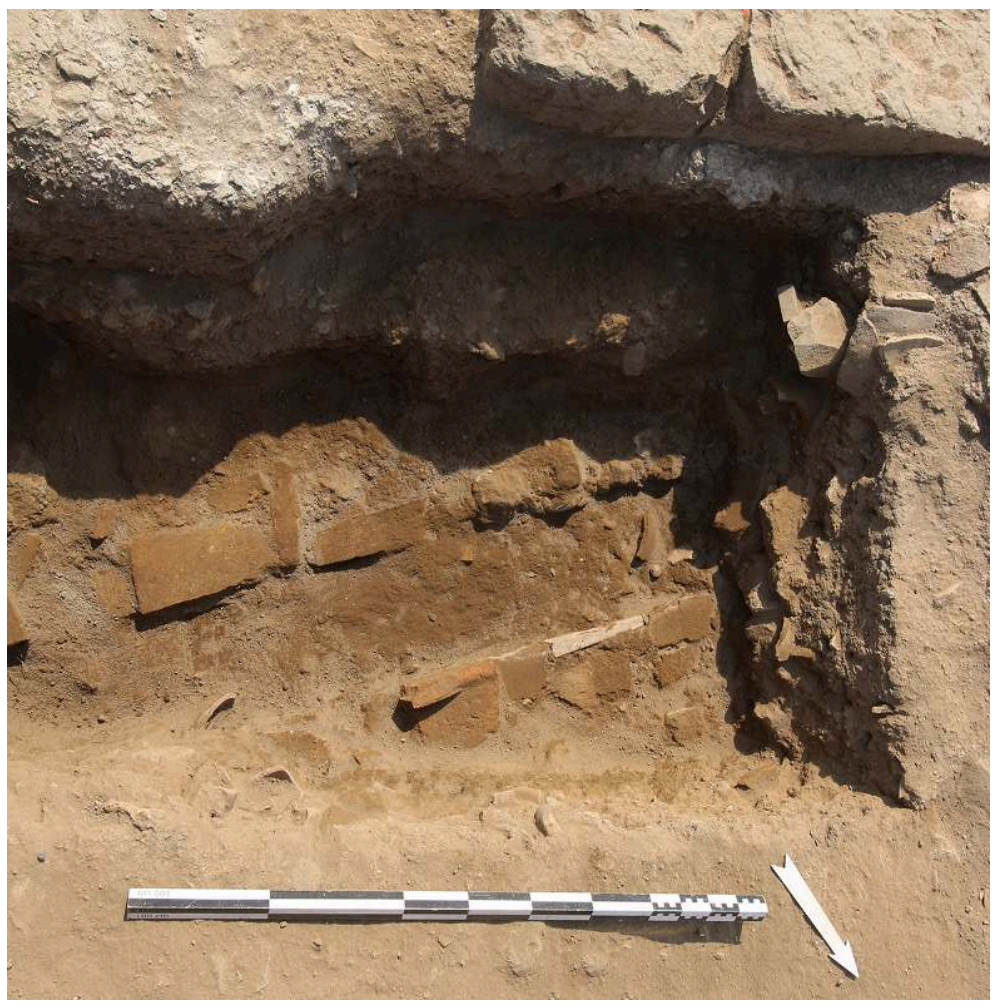

12 Dans la pièce B9 est apparu un sol en béton de tuileau portant des traces de peinture rouge (SO 676 ; fig. 11) sous le remblai d'abandon de la pièce (US 583).

Figure 11 - Le sol maçonné SO 676 de la pièce B9 (H. Hupfer).

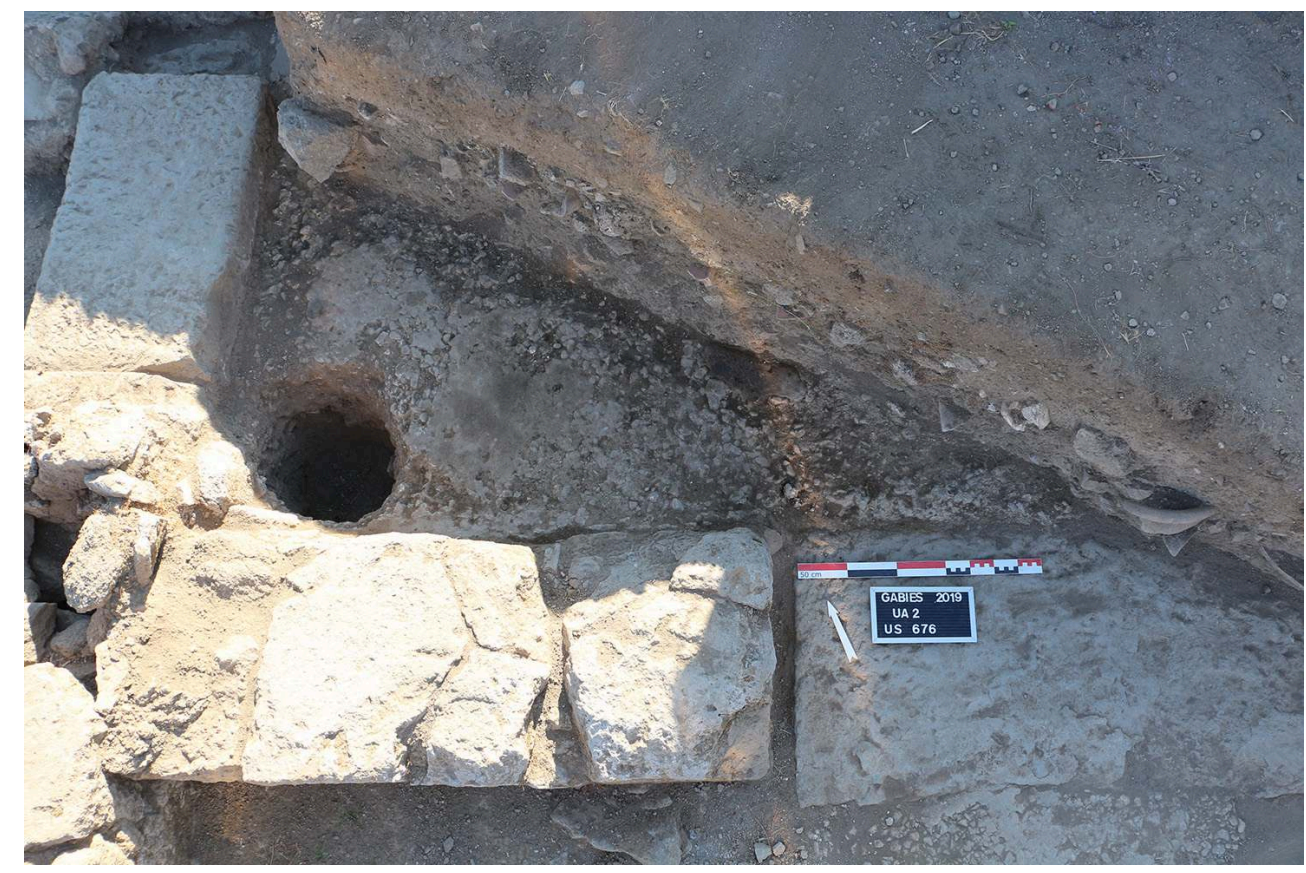




\section{La voirie}

Le trottoir sud de la rue est-ouest (VO 1) qui borde la domus UA 2 a été dégagé jusqu'à l'intersection avec la rue dallée en basalte d'axe nord-sud (VO 2). L'extrémité est du trottoir présente ainsi une bordure en arrondie qui se place au droit de l'angle nord-est du bâtiment (fig. 12). La bordure US 425 mesure ainsi 12,60 m de long depuis la bordure transversale US 554 jusqu'au carrefour et le dallage US 426, à l'est du seuil SO 427 , 5,50 m. Un bloc de basalte est posé de chant contre le mur de façade de la domus UA 2, au bout du trottoir, à l'est. Au total le trottoir sud mesure 20,35 m de long.

Figure 12 - Le croisement des rues VO 1 et VO 2 (P. Broutin).

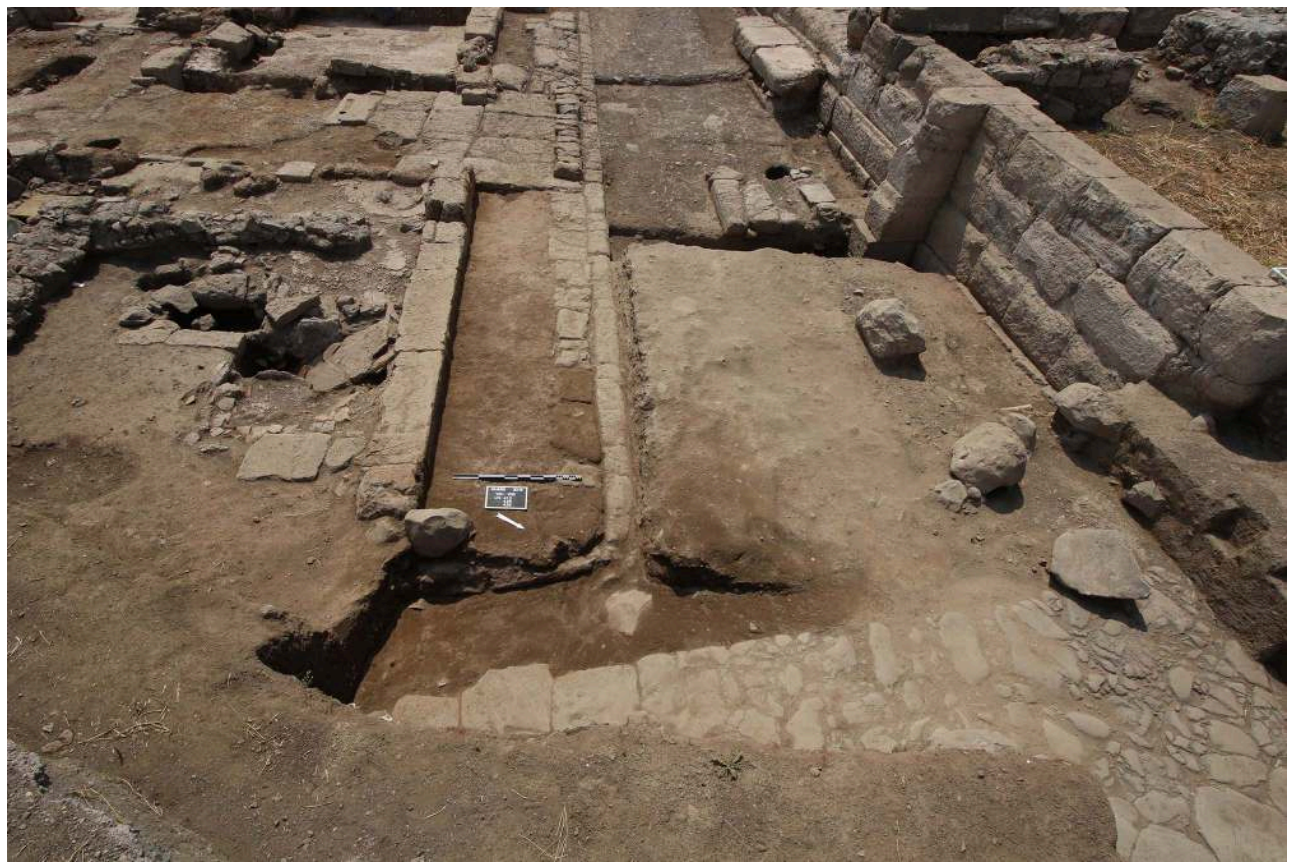

\section{L'angle sud-est du sanctuaire : le secteur du théâtre}

Dans le secteur A (fig. 13), l'écrêtement de la berme nord a été l'occasion de dégager toute la partie supérieure du mur de soutènement de la cavea (MR 604). Ce mur est en grand appareil rectangulaire isodome imparfait et conservé sur cinq assises mesurant $0,55 \mathrm{~m}$ de hauteur (fig. 14). Il présente un étagement en escalier de l'est vers l'ouest. Les blocs de l'assise supérieure conservent encore des bossages assez rustiques et plusieurs blocs présentent des encoches centrales plates ou circulaires, probablement pour des pinces de lavage. Dans sa partie ouest, le mur possède une assise de fondation supplémentaire reposant sur le banc rocheux tandis qu'à l'est, l'assise inférieure est posée sur cailloutis compact de lapis Gabinus mêlé à de la terre. Le lit d'attente de l'assise située directement face au mur MR 463 offre un pan incliné de 3,10 m de long constituant le sommier d'une voûte (fig. 15). MR 463 correspond de ce fait au piédroit sud d'une voûte en grand appareil couvrant un petit couloir de 2,80 $\mathrm{m}$ de large (espace A6) donnant accès à l'orchestra située à l'ouest. 
Figure 13 - Plan général de l'UA 1.

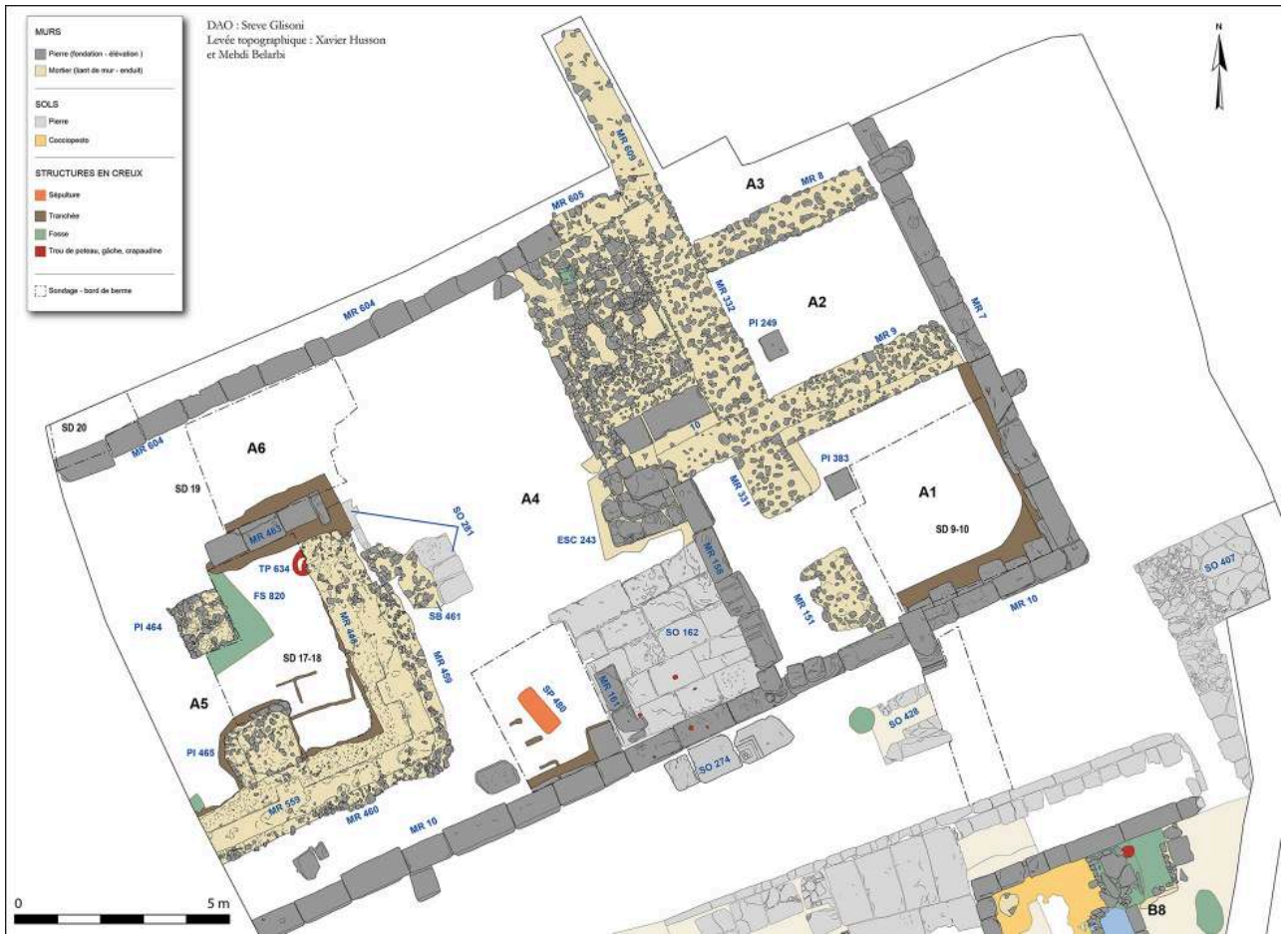

Figure 14 - Le mur de soutènement des gradins de la cavea (MR 604 à l'arrière-plan) et le piédroit MR 463 (A. Valois).

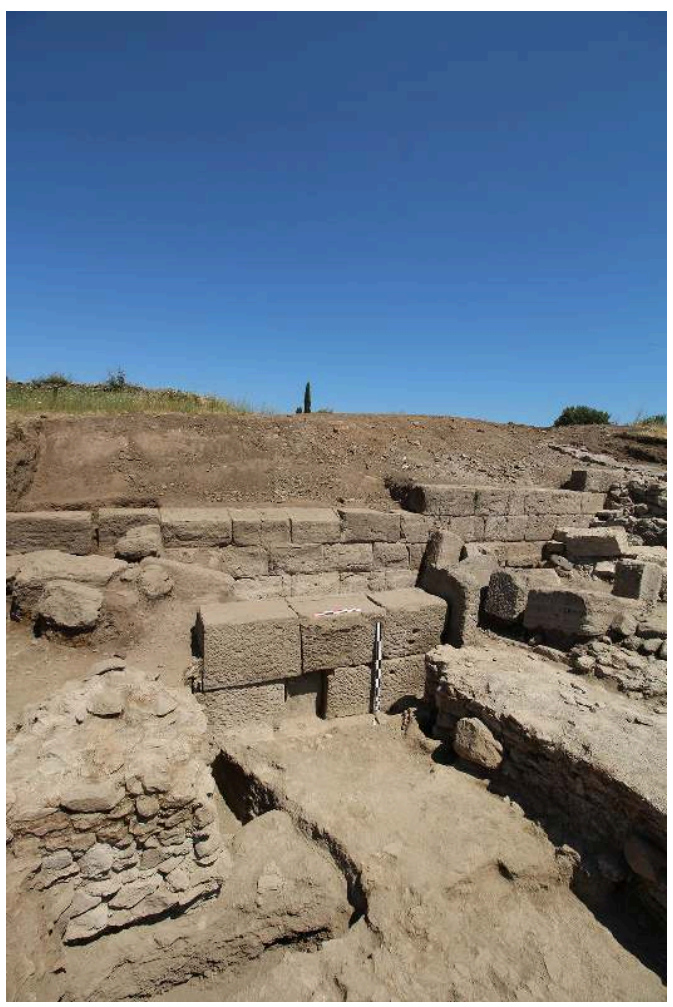


Figure 15 - Modélisation tridimensionnelle de l'espace A6 (M. Belarbi).

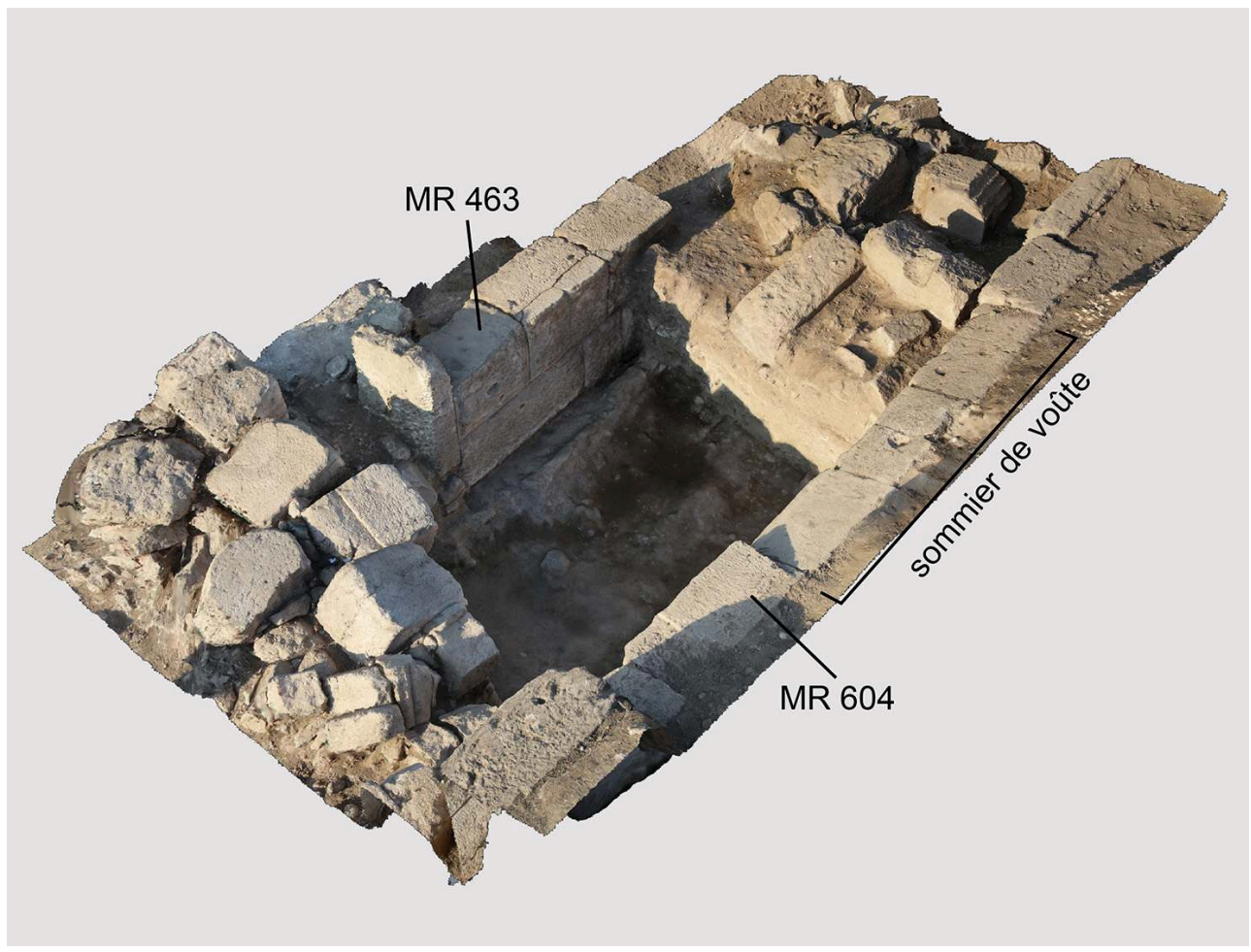

Contre ce mur a été mis au jour le tronçon d'une canalisation (CN 823) constituée d'un bloc de lapis Gabinus creusé mesurant $0,45 \mathrm{~m}$ de large et $0,22 \mathrm{~m}$ d'épaisseur; le bloc est brisé en deux et une partie se trouve dans l'US 236 (niveau d'effondrement) tandis que la partie encore en place est intégrée au massif de l'escalier ESC 243 (fig. 16). Le creusement ou canal d'écoulement adopte un profil en $\mathrm{U}$ et mesure 0,24 $\mathrm{m}$ de large pour $0,10 \mathrm{~m}$ de profondeur. La canalisation est recouverte par une dalle de couverture en lapis Gabinus mesurant 0,45 $\mathrm{m}$ de large et 0,11 m d'épaisseur. Son sens d'écoulement va de l'est vers l'ouest. 
Figure 16 - La canalisation CN 823 contre le mur de soutènement MR 604 (A. Valois).

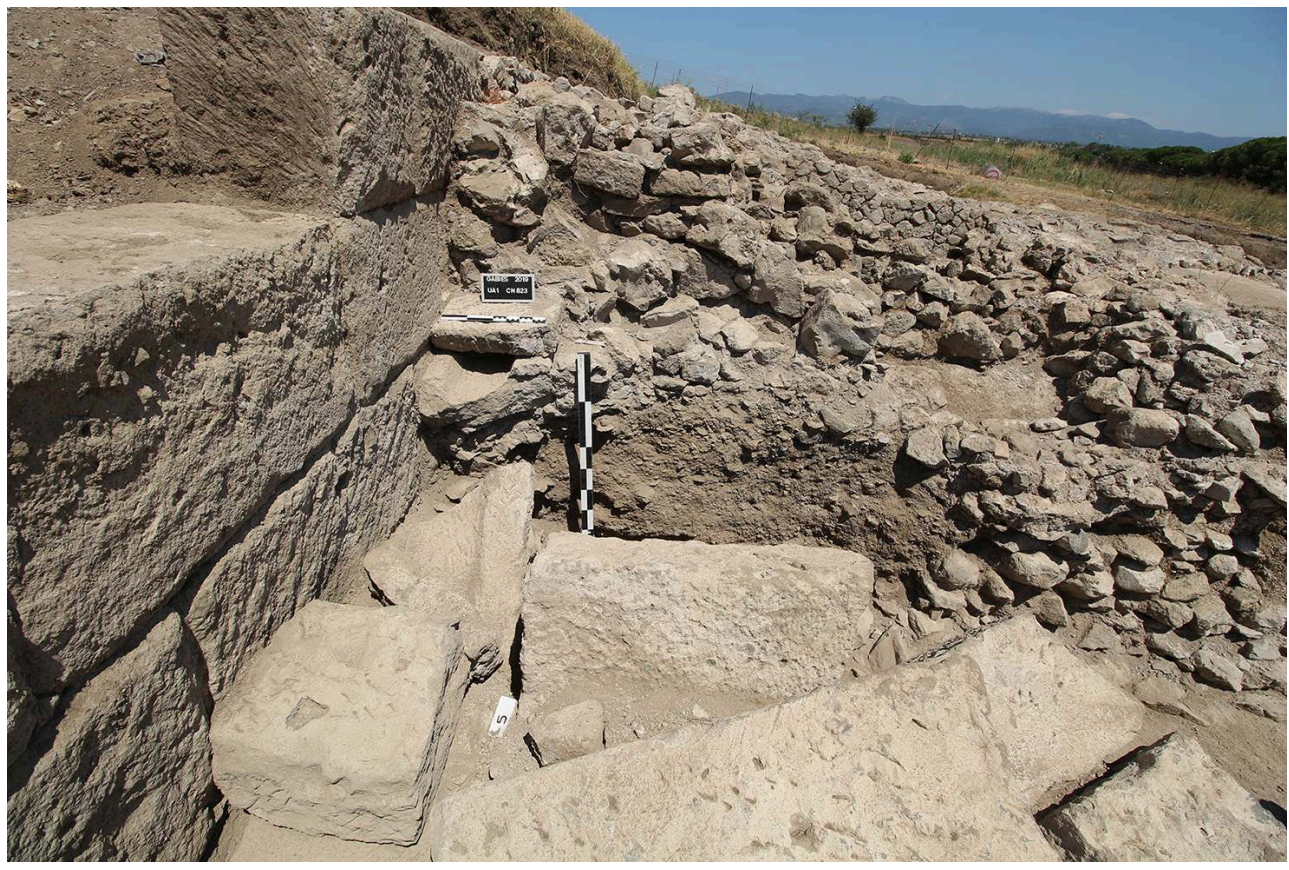

16 La fouille du passage latéral oriental situé entre la scène et la cavea a vu la découverte d'un bloc portant un fragment d'inscription que le support et la paléographie permettent de dater de la première moitié du $\mathrm{I}^{\mathrm{er}} \mathrm{s}$. av. J.-C. Cette inscription est hélas très lacunaire mais sa position in situ dans un niveau d'effondrement en place dans le bâtiment de scène, implique qu'elle est rattachée au complexe théâtral. Il pourrait de ce fait s'agir d'une dédicace liée à une phase de réfection du sanctuaire. Dans l'état actuel de nos connaissances cela ne demeure qu'une hypothèse.

17 Ce bloc fait partie d'un niveau d'effondrement contenant aussi des éléments de la voûte. Sous ce niveau a été mis au jour une couche de terre damée, vraisemblablement une préparation de sol destinée à accueillir un dallage qui a été totalement récupéré. Par ailleurs, l'absence de gradins dans un sondage stratigraphique manuel réalisé derrière le mur (fig. 17), en partie basse, a montré que ceux-ci n'étaient pas conservés dans ce secteur. 
Figure 17 - Sondage réalisé derrière le mur de soutènement MR 604 (A. Valois).

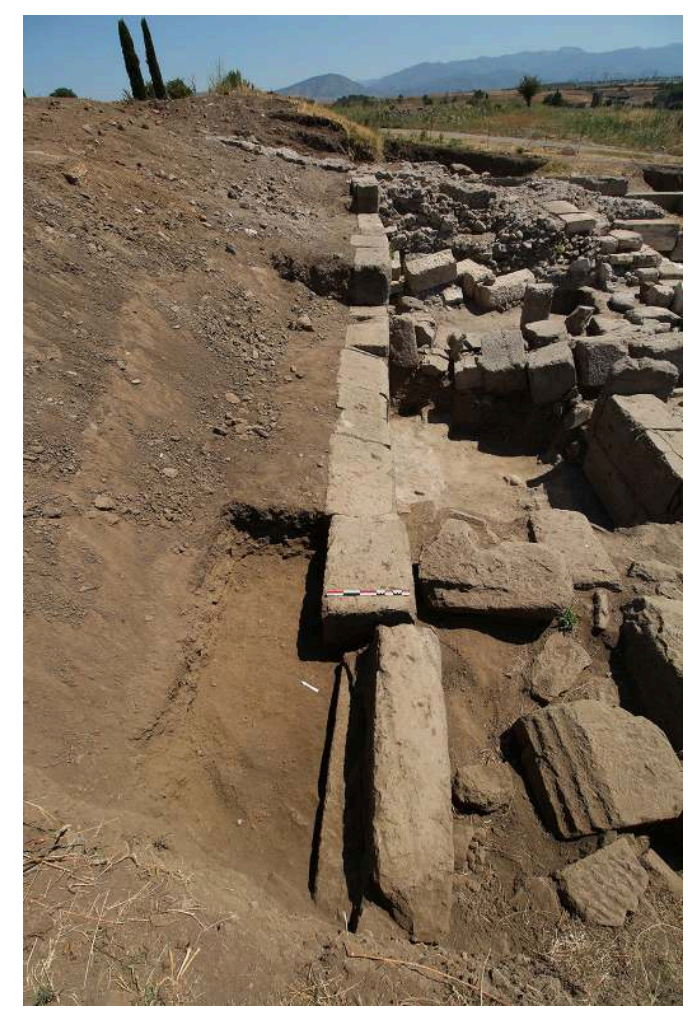

\section{Conclusion}

La campagne de fouille de 2019 a permis d'achever la fouille des pièces de la domus à l'exception des pièces B2 et B8. Il sera désormais nécessaire d'étendre l'emprise de fouille vers le sud et l'ouest afin d'en compléter le plan. Dans le secteur du théâtre, cette campagne nous permet de mieux préciser la chronologie et les modes de construction de l'édifice de scène. À l'avenir il faudra à la fois décaper vers l'ouest et le nord et procéder au grutage des blocs architecturaux monumentaux effondrés pour faciliter la fouille des niveaux sous-jacents.

\section{BIBLIOGRAPHIE}

Glisoni - Hasselin-Rous - Roger 2017 = S. Glisoni, I. Hasselin-Rous, D. Roger, Gabies. Campagnes 2014 et 2016 du Musée du Louvre et de la Surintendance de Rome, dans Chronique des activités archéologiques de l'École française de Rome [en ligne], 2017, http://journals.openedition.org/cefr/ 1644.

Glisoni - Hasselin - Roger 2018 = S. Glisoni, I. Hasselin, D. Roger, Gabies. Campagne 2017 du musée du Louvre et de la Surintendance de Rome, dans Chronique des activités archéologiques de l'École française de Rome [en ligne], 2018, http://journals.openedition.org/cefr/1905. 
Glisoni - Zanella 2019 = S. Glisoni, S. Zanella, Gabies. Campagne de 2018 du musée du Louvre et de la Surintendance de Rome, dans Chronique des activités archéologiques de l'École française de Rome [En ligne], 2019, http://journals.openedition.org/cefr/3980.

Roger - Sauvin - Taiuti 2014 = D. Roger, C.-É. Sauvin, A. Taiuti, Gabies, dans Chronique des activités archéologiques de l'École française de Rome [En ligne], 2014, http://cefr.revues.org/1151.

\section{NOTES}

1. L'Unité Architecturale 1 correspondant à l'angle sud-est du théâtre du sanctuaire de Junon Gabina.

2. L'Unité Architecturale 2 correspondant aux vestiges d'une domus à atrium d'époque républicaine.

3. Le seuil SO 500 mis au jour en 2017 se place face à cette entrée

4. Cet espace ne forme qu'une seule et même pièce à l'origine avant la construction des murs MR 569 et 576.

5. Pour des raisons évidentes de sécurité il n'a pas été possible de descendre plus bas.

\section{INDEX}

institutions Musée du Louvre - Département des antiquités grecques, étrusques et romaines ; Soprintendenza Speciale Archeologia, Belle Arti e Paesaggio di Roma - MIBACT ; Soprintendenza Archeologia, Belle Arti e Paesaggio per l'Area Metropolitana di Roma, la Provincia di Viterbo e l'Etruria Meridionale ; Institut national de recherches archéologiques préventives ; École française de Rome

Mots-clés : Gabies, domus, sanctuaire, Junon Gabina, cité romaine, urbanisme, théâtre

\section{AUTEUR}

\section{STEVE GLISONI}

Département des antiquités grecques, étrusques et romaines du Musée du Louvre - INRAP, UMR 7041 ArScan - steve.glisoni@louvre.fr 